\title{
THE CLASSIFICATION OF HYPERELLIPTIC THREEFOLDS
}

\author{
FABRIZIO CATANESE AND ANDREAS DEMLEITNER
}

\begin{abstract}
We complete the classification of hyperelliptic threefolds, describing in an elementary way the hyperelliptic threefolds with group $D_{4}$. These are algebraic and form an irreducible 2-dimensional family.
\end{abstract}

\section{INTRODUCTION}

A Generalized Hyperelliptic Manifold $X$ is defined to be a quotient $X=T / G$ of a complex torus $T$ by the free action of a finite group $G$ which contains no translations. We say that $X$ is a Generalized Hyperelliptic Variety if moreover the torus $T$ is projective, i.e., it is an Abelian variety $A$.

The main purpose of the present paper is to complete the classification of the Generalized Hyperelliptic Manifolds of complex dimension three. The cases where the group $G$ is Abelian were classified by H. Lange in [La01, using work of Fujiki Fu88 and the classification of the possible groups $G$ given by Uchida and Yoshihara in UY76]: the latter authors showed that the only possible non Abelian group is the dihedral group $D_{4}$ of order 8 .

This case was first excluded but it was later found that it does indeed occur (see CD18 for an account of the story and of the role of the paper [DHS08]). Our paper is fully self-contained and show that the family described in [CD18, gives all the possible hyperelliptic threefolds with group $D_{4}$.

Our main theorem is the following

Theorem 0.1. Let $T$ be a complex torus of dimension 3 admitting a fixed point free action of the dihedral group

$$
G:=D_{4}:=\left\langle r, s \mid r^{4}=1, s^{2}=1,(r s)^{2}=1\right\rangle,
$$

such that $G=D_{4}$ contains no translations.

Then $T$ is algebraic. More precisely, there are two elliptic curves $E, E^{\prime}$ such that:

(I) $T$ is a quotient $T:=T^{\prime} / H, H \cong \mathbb{Z} / 2$, where

$$
\begin{gathered}
T^{\prime}:=E \times E \times E^{\prime}=: E_{1} \times E_{2} \times E_{3}, \\
H:=\langle\omega\rangle, \omega:=(h+k, h+k, 0) \in T^{\prime}[2],
\end{gathered}
$$

and $h, k$ are 2-torsion element $h, k \in E[2]$, such that $h, k \neq 0, h+k \neq 0$;

Date: December 27, 2018.

AMS Classification: 14K99, 14D99, 32Q15

The present work took place in the framework of the ERC Advanced grant n. 340258, 'TADMICAMT'. 
(II) there is an element $h^{\prime} \in E^{\prime}$ of order precisely 4, such that, for $z=$ $\left(z_{1}, z_{2}, z_{3}\right) \in T^{\prime}$ :

$$
\begin{aligned}
& r(z)=\left(z_{2},-z_{1}, z_{3}+h^{\prime}\right)=R\left(z_{1}, z_{2}, z_{3}\right)+\left(0,0, h^{\prime}\right), \\
& s(z)=\left(z_{1}+h,-z_{2}+k,-z_{3}\right)=S\left(z_{1}, z_{2}, z_{3}\right)+(h, k, 0) .
\end{aligned}
$$

Conversely, the above formulae give a fixed point free action of the dihedral group $G=D_{4}$ which contains no translations.

In particular, we have the following normal form:

$$
\begin{gathered}
E=\mathbb{C} /(\mathbb{Z}+\mathbb{Z} \tau), \quad E^{\prime}=\mathbb{C} /\left(\mathbb{Z}+\mathbb{Z} \tau^{\prime}\right), \quad \tau, \tau^{\prime} \in \mathcal{H}:=\{z \in \mathbb{C} \mid \operatorname{Im}(z)>0\}, \\
h=1 / 2, k=\tau / 2, h^{\prime}=1 / 4 \\
r\left(z_{1}, z_{2}, z_{3}\right):=\left(z_{2},-z_{1}, z_{3}+1 / 4\right) \\
s\left(z_{1}, z_{2}, z_{3}\right):=\left(z_{1}+1 / 2,-z_{2}+\tau / 2,-z_{3}\right) .
\end{gathered}
$$

In particular, the Teichmüller space of hyperelliptic threefolds with group $D_{4}$ is isomorphic to the product $\mathcal{H}^{2}$ of two upper halfplanes.

\section{Proof of the MAIN TheOrem}

We use the following notation: $T=V / \Lambda$ is a complex torus of dimension 3 , which admits a free action of the group

$$
G=\left\langle r, s \mid r^{4}=s^{2}=(r s)^{2}=1\right\rangle \cong D_{4},
$$

such that the complex representation $\rho: G \rightarrow \mathrm{GL}(3, \mathbb{C})$ is faithful.

A first observation is that the complex representation $\rho$ of $G$ must contain the 2-dimensional irreducible representation $V_{1}$ of $G$ (else, $\rho$ would be a direct sum of 1-dimensional representations: this, by the assumption on the faithfulness of $\rho$, would imply that $G$ is Abelian, a contradiction).

Hence we have a splitting

$$
V=V_{1} \oplus V_{2},
$$

where $V_{2}$ is 1-dimensional, and we can choose an appropriate basis so that, setting $R:=\rho(r), S:=\rho(s)$, we are left with the two cases

$$
\begin{aligned}
& \text { Case 1: } R=\left(\begin{array}{ccc}
0 & 1 & \\
-1 & 0 & \\
& & 1
\end{array}\right), \quad S=\left(\begin{array}{ccc}
1 & & \\
& -1 & \\
& & -1
\end{array}\right), \\
& \text { Case 2: } R=\left(\begin{array}{ccc}
0 & 1 & \\
-1 & 0 & \\
& & 1
\end{array}\right), \quad S=\left(\begin{array}{lll}
1 & & \\
& -1 & \\
& & 1
\end{array}\right) .
\end{aligned}
$$

which are distinguished by the multiplicity of the eigenvalue 1 of $S$.

Indeed $R$ is necessarily of the form above, since the freeness of the $G$-action implies that $\rho(g)$ must have eigenvalue 1 for every $g \in G$.

Lemma 1.1. In both Cases 1 and 2, the complex torus $T=V / \Lambda$ is isogenous to a product of three elliptic curves, $T \sim_{i s o g} . E_{1} \times E_{2} \times E_{3}$, where $E_{i} \subset T$, for $i=1,2,3$ and $E_{1}$ and $E_{2}$ are isomorphic elliptic curves. In other words, writing $E_{j}=W_{j} / \Lambda_{j}$, the complex torus $T$ is isomorphic to

$$
\left(E_{1} \times E_{1} \times E_{3}\right) / H, \quad H=\Lambda /\left(\Lambda_{1} \oplus \Lambda_{2} \oplus \Lambda_{3}\right) .
$$


Proof. Let $I$ be the identity of $T$.

In Case 1 , we set $E_{1}:=\operatorname{ker}(S-I)^{0}=\operatorname{im}(S+I), E_{3}:=\operatorname{ker}(R-I)^{0}$ and $E_{2}:=R\left(E_{1}\right)$ (here, the superscript zero denotes the connected component of the identity). Then it is clear that $E_{1} \cong E_{2}$, and that $T$ is isogenous to $E_{1} \times E_{2} \times E_{3}$.

In Case 2, we define similarly $E_{2}:=\operatorname{ker}(S+I)^{0}=\operatorname{im}(S-I), E_{3}:=\operatorname{ker}(R-$ $I)_{0}$ and $E_{1}:=R\left(E_{2}\right)$. We obtain again $E_{1} \cong E_{2}$, and that $T$ is isogenous to $E_{1} \times E_{2} \times E_{3}$.

Lemma 1.2. Writing $E_{j}=W_{j} / \Lambda_{j}$, the following statements hold.

(1) In Case 1, the lattice $\Lambda_{2}$ is equal to $W_{2} \cap \Lambda$.

(2) In Case 2, the lattice $\Lambda_{1}$ is equal to $W_{1} \cap \Lambda$.

Proof. (1) Obviously, $E_{2}=R\left(E_{1}\right)=W_{2} / R\left(\Lambda_{1}\right)$, i.e., $\Lambda_{2}=R\left(\Lambda_{1}\right) \subset W_{2} \cap \Lambda$. On the other hand, $R\left(W_{2} \cap \Lambda\right) \subset W_{1} \cap \Lambda=\Lambda_{1}$, and applying the automorphism $R$ of $\Lambda$ gives $W_{2} \cap \Lambda \subset R\left(\Lambda_{1}\right)=\Lambda_{2}$.

(2) Here, $E_{1}=R\left(E_{2}\right)=W_{1} / R\left(\Lambda_{2}\right)$, i.e., $\Lambda_{1}=R\left(\Lambda_{2}\right) \subset W_{1} \cap \Lambda$. For the converse inclusion, observe $R\left(W_{1} \cap \Lambda\right) \subset W_{2} \cap \Lambda=\Lambda_{2}$, and applying $R$ yields again the result.

We can now choose coordinates on $V$ such that $r$ is induced by a transformation of the form

$$
r\left(z_{1}, z_{2}, z_{3}\right)=\left(z_{2},-z_{1}, z_{3}+c_{3}\right),
$$

by choosing as the origin in $V_{1}$ a fixed point of the restriction of $r$ to $V_{1}$.

We can now view $r, s$ as affine self maps of $T$ induced by affine self maps of $E_{1} \times E_{2} \times E_{3}$ of the form

$$
\begin{gathered}
r\left(z_{1}, z_{2}, z_{3}\right)=\left(z_{2},-z_{1}, z_{3}+c_{3}\right), \\
s\left(z_{1}, z_{2}, z_{3}\right):=\left(z_{1}+a_{1},-z_{2}+a_{2}, \pm z_{3}+a_{3}\right),
\end{gathered}
$$

and sending the subgroup $H$ to itself.

Lemma 1.3. The freeness of the action of the powers of $r$ is equivalent to: $H$ contains no element with last coordinate equal to $c_{3}$, or $2 c_{3}$.

Moreover, $\left(0,0,4 c_{3}\right) \in H$.

Proof. $r(z)=z$ is equivalent to $\left(z_{1}-z_{2}, z_{1}+z_{2},-c_{3}\right) \in H$. However, the endomorphism

$$
\left(z_{1}, z_{2}\right) \mapsto\left(z_{1}-z_{2}, z_{1}+z_{2}\right)
$$

of $E_{1} \times E_{2}$ is surjective, hence $H$ cannot contain any element with last coordinate equal to $c_{3}$.

Since $r^{2}(z)=\left(-z_{1},-z_{2}, z_{3}+2 c_{3}\right), r^{2}(z)=z$ is equivalent to $\left(-2 z_{1},-2 z_{2}, 2 c_{3}\right) \in$ $H$, and we reach the similar conclusion that $H$ cannot contain any element with last coordinate equal to $2 c_{3}$.

Finally, the condition that $r^{4}$ is the identity is equivalent to $\left(0,0,4 c_{3}\right) \in H$.

Proposition 1.1. Case 2 does not occur. 
Proof. Since we assume that

$$
s\left(z_{1}, z_{2}, z_{3}\right):=\left(z_{1}+a_{1},-z_{2}+a_{2}, z_{3}+a_{3}\right),
$$

and that $s^{2}$ is the identity, it must be

$$
\left(2 a_{1}, 0,2 a_{3}\right) \in H .
$$

Consider now rs:

$$
r s(z)=\left(-z_{2}+a_{2},-z_{1}-a_{1}, z_{3}+a_{3}+c_{3}\right) .
$$

The condition that $(r s)^{2}$ is the identity is equivalent to:

$$
\left(a_{1}+a_{2},-\left(a_{1}+a_{2}\right), 2\left(a_{3}+c_{3}\right)\right) \in H .
$$

This condition, plus the previous one, imply that

$$
\left(a_{2}-a_{1},-\left(a_{1}+a_{2}\right), 2 c_{3}\right) \in H,
$$

contradicting Lemma 1.3 .

Henceforth we shall assume that we are in Case 1, and we can choose the origin in $E_{3}$ so that

$$
s\left(z_{1}, z_{2}, z_{3}\right):=\left(z_{1}+a_{1},-z_{2}+a_{2},-z_{3}\right) .
$$

Lemma 1.4. If

$$
s\left(z_{1}, z_{2}, z_{3}\right):=\left(z_{1}+a_{1},-z_{2}+a_{2},-z_{3}\right),
$$

then

$$
\left(2 a_{1}, 0,0\right) \in H
$$

and $H$ contains no element of the form

$$
\left(a_{1}, w_{2}, w_{3}\right) \text {. }
$$

Proof. The first condition is equivalent to $s^{2}$ being the identity, while the second is equivalent to the condition that $s$ acts freely, since $s(z)=z$ is equivalent to $\left(a_{1},-2 z_{2}+a_{2},-2 z_{3}\right) \in H$.

Proposition 1.2. For each $\lambda \in \Lambda$ there exist $\lambda^{\prime} \in \Lambda, \lambda_{1} \in \Lambda_{1}, \lambda_{2} \in \Lambda_{2}, \lambda_{3} \in$ $\Lambda_{3}$, , such that

$$
2 \lambda=\lambda_{1}+\lambda^{\prime}, \quad 2 \lambda^{\prime}=\lambda_{2}+\lambda_{3}
$$

More precisely, we even have:

$$
\Lambda \subset(1 / 2) \Lambda_{1}+(1 / 2) \Lambda_{2}+(1 / 4) \Lambda_{3} .
$$

Proof. Let $\lambda \in \Lambda$ : we can write

$$
2 \lambda=\underbrace{(I+S) \lambda}_{=: \lambda_{1} \in \Lambda_{1}}+\underbrace{(I-S) \lambda}_{=: \lambda^{\prime} \in \Lambda} .
$$

Furthermore, since $\lambda^{\prime} \in \operatorname{im}(I-S)$, we obtain

$$
2 \lambda^{\prime}=\underbrace{\left(I+R^{2}\right) \lambda^{\prime}}_{=: \lambda_{3} \in \Lambda_{3}}+\underbrace{\left(I-R^{2}\right) \lambda^{\prime}}_{=: \lambda_{2} \in \Lambda \cap W_{2}=\Lambda_{2}} .
$$

Hence, $\lambda=\frac{\lambda_{1}}{2}+\frac{\lambda_{2}}{4}+\frac{\lambda_{3}}{4}$ for unique $\lambda_{j} \in \Lambda_{j}$. 
Applying the automorphism $R$ of $\Lambda$ and the unicity of the $\lambda_{j}$ yields the result, since $R$ exchanges $\Lambda_{1}$ and $\Lambda_{2}$.

Proposition 1.3. We have

$$
\Lambda \subset(1 / 2) \Lambda_{1}+(1 / 2) \Lambda_{2}+(1 / 2) \Lambda_{3} .
$$

Proof. For $\lambda \in \Lambda$ we can write $\lambda=\frac{\lambda_{1}}{2}+\frac{\lambda_{2}}{2}+\frac{\lambda_{3}}{4}$ for unique $\lambda_{j} \in \Lambda_{j}$.

We now use the property

$$
E_{i} \hookrightarrow T \Rightarrow \forall(0,0, d) \in H, d=0 .
$$

Indeed, $2 \lambda=\lambda_{1}+\lambda_{2}+\frac{\lambda_{3}}{2}$, hence $\left(0,0,\left[\frac{\lambda_{3}}{2}\right]\right) \in H$ and $\frac{\lambda_{3}}{2}=0$ in $E_{3}$. Equivalently, there is an element $\lambda_{3}^{\prime} \in \Lambda_{3}$ with

$$
\frac{\lambda_{3}}{4}=\frac{\lambda_{3}^{\prime}}{2}
$$

Lemma 1.5. Consider the transformation rs:

$$
r s(z)=\left(-z_{2}+a_{2},-z_{1}-a_{1},-z_{3}+c_{3}\right) .
$$

The condition that its square is the identity amounts to

$$
\left(a_{1}+a_{2},-\left(a_{1}+a_{2}\right), 0\right) \in H,
$$

while the freeness of its action is equivalent to the fact that $H$ contains no element of the form

$$
\left(w_{1}-a_{2}, w_{1}+a_{1}, w_{3}\right) \Leftrightarrow \forall\left(d_{1}, d_{2}, d_{3}\right) \in H: \quad d_{1}+a_{2} \neq d_{2}-a_{1} .
$$

Proof. The first condition is straighforward, while the freeness of the action is equivalent to the non existence of $\left(z_{1}, z_{2}, z_{3}\right)$ such that

$$
\left(z_{1}+z_{2}-a_{2}, z_{2}+z_{1}+a_{1}, 2 z_{3}-c_{3}\right) \in H .
$$

As usual, we observe that for each $w_{1}, w_{3}$ there exist $z_{1}, z_{2}, z_{3}$ with $z_{1}+z_{2}=$ $w_{1}, 2 z_{3}-c_{3}=w_{3}$.

We put together the conclusions of Lemmas 1.3, 1.4, 1.5,

- (i) $\left(0,0,4 c_{3}\right) \in H$

- (ii) $\left(2 a_{1}, 0,0\right) \in H$

- (iii) $\left(a_{1}+a_{2},-a_{1}-a_{2}, 0\right) \in H$, hence also $\left(a_{1}-a_{2}, a_{1}+a_{2}, 0\right) \in H$.

(1) $H$ contains no element of the form $\left(w_{1}, w_{2}, c_{3}\right)$,

(2) nor of the form $\left(w_{1}, w_{2}, 2 c_{3}\right)$

(3) nor of the form $\left(a_{1}, w_{2}, w_{3}\right)$

(4) nor of the form $\left(w_{1}, w_{2}, w_{3}\right)$ with $w_{1}+a_{2}=w_{2}-a_{1}$.

It follows from (iii) and (3) that $a_{2} \neq 0$. While the condition that each element of $H$ which has two coordinates equal to zero is indeed zero (since $E_{i}$ embeds in $T$ !) imply

$$
2 a_{1}=0,4 c_{3}=0 .
$$

By conditions (1), (2), (3) the elements $a_{1}, c_{3}$ have respective orders exactly 2, 4. Moreover: 
- (4) and (i) imply that $a_{1}+a_{2} \neq 0$

- (ii), (iii) and the fact that $H$ has exponent 2 implies $2 a_{2}=2 a_{1}=0$, $2 a_{1}+2 a_{2}=0$. Hence $a_{1} \neq a_{2}$ are nontrivial 2-torsion elements.

We have thus obtained the desired elements

$$
h:=a_{1}, k:=a_{2}, h^{\prime}:=c_{3} .
$$

It suffices to show that $H$ is generated by $\omega:=(h+k, h+k, 0)=\left(a_{1}+\right.$ $\left.a_{2}, a_{1}+a_{2}, 0\right)$.

Observe first that $\omega \in H$, by condition (iii).

Condition (4) implies that the first coordinate of an element of $H$ must be a multiple of $\left(a_{1}+a_{2}\right)$ : since it cannot equal $a_{1}$, by condition (3), and if it equals $a_{2}$, we can add $\omega$ and obtain an element of $H$ with first coordinate $a_{1}$. Using $R$, we infer that both coordinates must be a multiple of $\left(a_{1}+a_{2}\right)$. Possibly adding $\omega$, we may assume that $w_{1}=0$ : then by (4) we conclude that also $w_{2}=0$. Finally, the condition that each element of $H$ which has two coordinates equal to zero is indeed zero, show that $H$ is then generated by $\omega$, as we wanted to show.

The last assertions of the main theorem follow now in a straightforward way (see [CC17] concerning general properties of Teichmüller spaces of hyperelliptic manifolds).

\section{REFERENCES}

[CC17] F. Catanese, P. Convaja: Teichmüller spaces of generalized hyperelliptic manifolds. Complex and symplectic geometry, 39-49, Springer INdAM Ser., 21, Springer, Cham (2017).

[CD18] F. Catanese, A. Demleitner: Hyperelliptic Threefolds with group $D_{4}$, the Dihedral group of order 8. Preprint (2018), arXiv:1805.01835.

[DHS08] K. Dekimpe, M. HaŁenda, A. Szczepański: Kähler flat manifolds. J. Math. Soc. Japan 61 (2009), no. 2, 363-377.

[Fu88] A. FujIKI: Finite automorphism groups of complex tori of dimension two. Publ. Res. Inst. Math. Sci., 24 (1988), 1-97.

[La01] H. LAnge: Hyperelliptic varieties. Tohoku Math. J. (2) 53 (2001), no. 4, 491510.

[UY76] K. UCHIDA, H. YoshiHARA: Discontinuous groups of affine transformations of $\mathbb{C}^{3}$. Tohoku Math. J. (2) 28 (1976), no. 1, 89-94.

Lehrstuhl Mathematik VIII, Mathematisches Institut der Universität Bayreuth, NW II, Universitätsstr. 30, 95447 BAYREUth

E-mail address: Fabrizio.Catanese@uni-bayreuth.de

Andreas.Demleitner@uni-bayreuth.de 